\title{
SÍNTESIS DE ESPIROBRASINOESTEROIDES ANÁLOGOS DE LA 6-DESOXOCASTASTERONA
}

Martín A. Iglesias Arteaga, Vivian Leliebre Lara, Carlos Pérez Martínez, Francisco Coll Manchado*

Laboratório de Productos Naturales - Departamento de Química Orgánica - Facultad de Química - Universidad de la Habana Zapata y G. C. - Habana - 10400 - Cuba.

Recebido em 3/4/96; aceito em 30/10/96

\begin{abstract}
SYNTHESIS OF SPIROBRASSINOSTEROIDS ANALOGS OF THE 6-DESOXOCASTASTERONE. The synthesis of two new spirostanic analogs of the natural occurring brassinosteroid 6 desoxocastasterone (1) is described. The scheme consists in the formation and elimination of tigogenin mesylate followed by catalytic dihydroxylation of the resulting $\Delta^{2}$-steroid (3) and acetylation of the $2 \alpha, 3 \alpha$-diol introduced.Treatment diacetate (5) with $\mathrm{NaNO}_{2} / \mathrm{BF}_{3} . \mathrm{Et}_{2} \mathrm{O}$ and chromatography in alumina led to a 23 -keto (6) which on reduction produced the $23 \mathrm{~S}$ alcohol (8) as major product. Saponification of the $2 \alpha, 3 \alpha$-diacetoxy-23-keto compound (6) and the $2 \alpha, 3 \alpha$-diacetoxy-23hydroxy compound (8) led to the spirobrasinosteroids (7) and (9). ${ }^{13} \mathrm{C} N M R$ and ${ }^{1} \mathrm{H}$ RMN characteristics derived from substitution at $\mathrm{C}_{23}$ are briefly discussed.
\end{abstract}

Keywords: Spirobrassinosteroids; synthesis; RMN.

\section{INTRODUCCIÓN}

Los brasinoesteroides son compuestos naturales que al ser aplicados a las plantas en concentraciones que oscilan entre $10^{-2}$ y $10^{-4} \mu \mathrm{g} / \mathrm{ml}$ producen un marcado aumento en los ritmos del crecimiento vegetal, el aceleramiento de la maduración de la cosechas, así como el aumento de la resistencia a plagas y diferentes factores de "stress" como alta salinidad, sequía, bajas y altas temperaturas ${ }^{1}$. Estos compuestos, que han sido encontrados en todos los órganos de los representantes de diferentes familias del reino vegetal, marino y terrestre en que han sido buscados, son considerados por muchos especialistas como una nueva clase de hormona vegetal ${ }^{2}$. Algunos estudios que han dejado establecido los requerimientos estructurales para una potente actividad estimuladora del crecimiento vegetal, indican sin embargo que compuestos con cadenas laterales modificadas, presentan en determinados casos una buena actividad biológica ${ }^{3,4}$. Así, han sido sintetizados análogos de brasinoesteroides que presentan las funciones de los brasinoesteroides naturales en los anillos A y B y que carecen de cadena lateral o son portadores de cadenas laterales de tipo androstano ${ }^{5}$, pregnano 6 , colano $^{7}$, colestano ${ }^{8}$ con diferentes patrones de sustitución.

Recientemente han sido sintetizados, partiendo de diferentes sapogeninas esteroidales, varios análogos de brasinoesteroides portadores de la cadena lateral espirocetálica ${ }^{9}$. Estos análogos espirostánicos de brasinoesteroides o espirobrasinoesteroides han mostrado en determinados casos una potente actividad biológica ${ }^{10}$; lo que sugiere que la agrupación cetálica sobre la posición $\mathrm{C}_{22}$ de la cadena espirostánica produce efectos cualitativamente semejantes a los de la función alcohólica en $\mathrm{C}_{22}$ de la cadena lateral de los brasinoesteroides. Tomando en cuenta que en la posición $\mathrm{C}_{23}$ de la cadena lateral de los brasinoesteroides naturales hay presente una función alcohólica y por otra parte que se supone que algunos compuestos naturales, que su estructura no ha sido totalmente esclarecida, pueden ser portadores de funciones carbonílicas en la cadena lateral, hemos decidido realizar la síntesis de un grupo de espirobrasinoesteroides portadores de funciones alcohólicas o carbonílicas en la posición $\mathrm{C}_{23}{ }^{11}$ con el objetivo de evaluar que efecto ocasiona la funcionalización de esta posición, en la actividad estimuladora del crecimiento vegetal de los espirobrasinoesteroides. En este trabajo se presenta la síntesis, a partir de la sapogenina esteroidal tigogenina, de dos nuevos análogos de brasinoesteroides caracterizados por poseer una cadena lateral espirocetálica portadora de las mencionadas funciones en la posición $\mathrm{C}_{23}$, además de presentar un núcleo esteroidal semejante al del brasinoesteroide natural 6-desoxocastasterona (1). Se ofrecen detalles sobre la caracterización espectroscópica basada en los espectros IR-FT y RMN de los compuestos obtenidos.

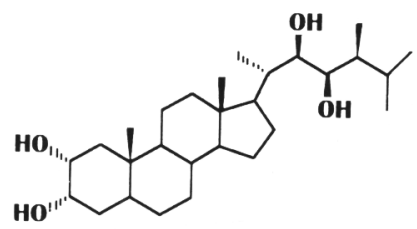

Figura 1. Estructura de la 6-desoxocastasterona (1).

\section{DISCUSIÓN DE RESULTADOS}

La tigogenina (2) sapogenina esteroidal que puede ser fácilmente obtenida en nuestro país, presenta las funciones adecuadas para realizar con buen rendimiento la introducción del sistema diol presente en el anillo A de la 6-desoxocastasterona. El esquema de trabajo seguido (Figura 2) que reproduce inicialmente los pasos reportados para la preparación del diol $(4)^{9}$, análogo espirostánico del compuesto natural (1), incluye la formación y eliminación de un éster sulfónico de la función hidroxílica $3 \beta$ presente en el anillo A, para producir la olefina (3) a partir de la cual se obtiene el citado análogo (4) por tratamiento con $\mathrm{OsO}_{4}$ en presencia de óxido de $\mathrm{N}$-metilmorfolina.

La acetilación del diol (4) produce el diacetato (5) que disuelto en ácido acético glacial, al ser tratado con trifluoruro de boro eterato y nitrito de sodio y posteriormente ser sometido a una cromatografia en columna de alúmina de actividad Brockman III, da lugar a la cetona (6) con un rendimiento del $69 \%$ que se corresponde con los reportes de aplicación de este método a otras sapogeninas esteroidales ${ }^{12,13}$. La reducción de la cetona (6) con borhidruro de sodio produce, según indicó el control cromatográfico, una mezcla del (23S,25R)-2 $\alpha, 3 \alpha-$ diacetoxi-5 $\alpha$-espirostan-23-ol (8) (ecuatorial) y su isómero $23 \mathrm{R}$ (axial) que fueron separados produciéndose un $85 \%$ del diacetato (8). Las reducciones de cetonas esteroidales utilizando hidruros metálicos han sido examinadas por diferentes autores ${ }^{14}$ y en el 


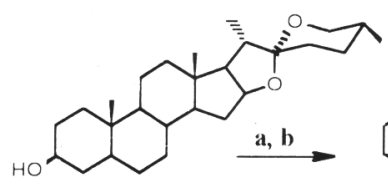

(2)

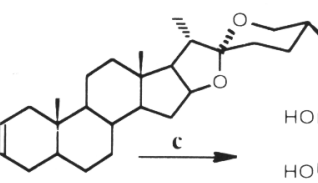

(3)

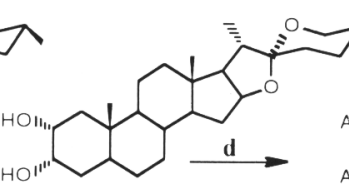

(4)

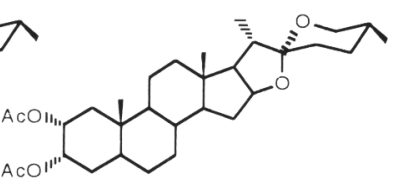

(5)

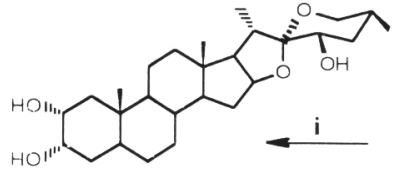

(9)

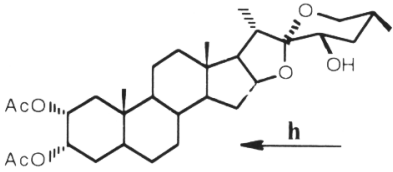

(8)

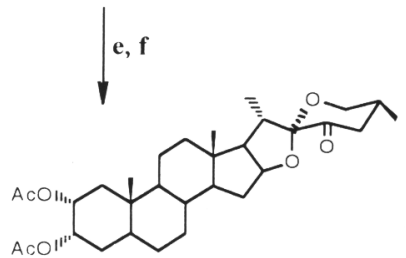

(6) a) $\mathrm{CH}_{3} \mathrm{SO}_{2} \mathrm{Cl} /$ piridina

b) $\mathrm{LiBr} / \mathrm{Li}_{2} \mathrm{CO}_{3} / \mathrm{DMF}$ reflujo

c) $\mathrm{OsO}_{4} / \mathrm{ONMM} / \mathrm{THF}$

d) $\mathrm{Ac}_{2} \mathrm{O} /$ piridina

c) $\mathrm{BF}_{3} \cdot \mathrm{Et}_{2} \mathrm{O} / \mathrm{CH}_{3} \mathrm{COOH} / \mathrm{NaNO}$ f) columna de alumina actividad III
g) $\mathrm{KOH} / \mathrm{MeOH}$
h) $\mathrm{NaBH} / \mathrm{MeOH}$
i) $\mathrm{KOH} / \mathrm{MeOH}$

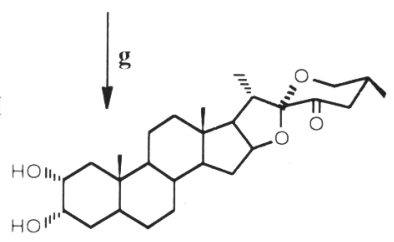

(7)

Figura 2. Esquema de síntesis seguido.

caso del borhidruro de sodio, se reconoce una marcada sensibilidad a efectos estéricos que limiten su acercamiento al sitio de reacción. Esta sensibilidad es especialmente alta cuando la reacción se realiza en presencia de solventes capaces de sustituir hidruros para dar lugar a alcóxidos de boro de volumen considerable ${ }^{15,16}$. La presencia de átomos de hidrógeno con orientación espacial $\alpha$ axial enlazados a $\mathrm{C}_{24}$ y $\mathrm{C}_{26}$ (Figura 3) limita el acercamiento $\alpha$ a la función carbonílica, proceso que puede considerarse impedido además, por la cercanía del átomo de oxígeno integrante del puente etéreo entre $\mathrm{C}_{16}$ y $\mathrm{C}_{22}$, quedando reprimida así la producción del isómero 23R (axial). No existen elementos que impidan apreciablemente el acercamiento $\beta$ de la molécula del reductor y por consiguiente la producción del alcohol ecuatorial 23S. La saponificación de los diacetatos (6) y (8) produjo los análogos espirostánicos de la 6-desoxocastasterona funcionalizados en $\mathrm{C}_{23}$ (7) y (9) con rendimientos totales de $35 \%$ y $28 \%$ respectivamente.

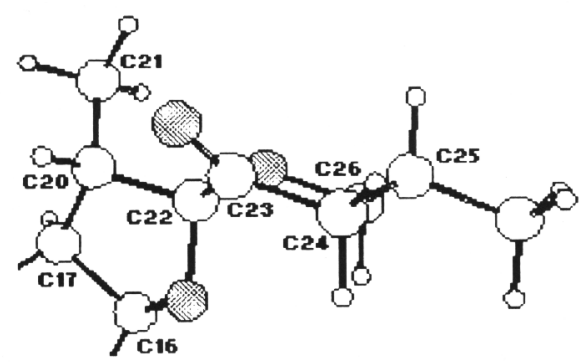

Figura 3. Gráfico molecular de la cadena lateral de una 23cetosapogenina esteroidal.

Las características espectroscópicas derivadas de la introducción del sistema $2 \alpha, 3 \alpha$-diol en el anillo A se corresponden con las previamente reportadas ${ }^{9}$ y no se ven afectadas por la modificación de la cadena lateral. La introducción de las agrupaciones alcohólica y carbonílica en la posición $\mathrm{C}_{23}$ produce la desaparición del sistema de bandas característico del espectro IR de la agrupación espirocetálica y puede ser reconocida por la presencia de las bandas correspondientes a las vibraciones de valencia de los grupos introducidos. El valor algo alto de la vibración de valencia carbonílica puede ser atribuido a la presencia en posición $\alpha$ del carbono espirocetálico $\mathrm{C}_{22}$ unido a dos átomos de oxígeno.

La presencia de una función carbonílica en la posición $\mathrm{C}_{23}$ ocasiona marcados cambios en los corrimientos químicos asociados a los protones situados en la cadena lateral. La inversión de las posiciones relativas y el desblindaje de las señales de H-26 axial y H-26 ecuatorial constituyen índices de la presencia de la función carbonílica en $\mathrm{C}_{23}$. Adicionalmente el desblindaje de los protones H-20,H-24, H-25 y H-27 caracteriza la transformación realizada. Estas afectaciones pueden ser atribuidas básicamente a la anisotropía magnética de la función introducida, aunque en el caso de la señal correspondiente al protón H-20 es posible considerar adicionalmente interacciones estéricas con el oxígeno carbonílico. El espectro protónico de los compuestos 23-hidroxilados se caracteriza por la presencia de la señal desblindada del protón carbinólico $\mathrm{H}-23$ que aparece superpuesta con la correspondiente a H-26 ecuatorial. Se destaca el desblindage del protón $\mathrm{H}-20$ atribuible a una interacción $\delta$ syn con el grupo hidroxilo.

En el espectro RMN ${ }^{13} \mathrm{C}$ la introducción de una función carbonílica en la cadena lateral puede reconocerse por la nueva señal correspondiente a $\mathrm{C}_{23}$. Es significativo el desblindaje de las posiciones $\mathrm{C}_{24}$ y $\mathrm{C}_{25}$, atribuible respectivamente al efecto de la sustitución en $\beta$ y a la pérdida de la interacción blindante con el protón $\mathrm{H}-23$ que supone el tránsito de $\mathrm{C}_{23}$ de hibridación $\mathrm{sp}_{3}$ a $\mathrm{sp}_{2}$. Adicionalmente, la presencia del sustituyente en $\mathrm{C}_{23}$ produce el blindaje de $\mathrm{C}_{20}$ que puede ser atribuido a una interacción blindante $\gamma$ gauche. La presencia de la función hidroxílica ecuatorial en $\mathrm{C}_{23}$ se caracteriza por la aparición de una nueva señal funcional correspondiente al carbinol generado y produce el desblindaje de $\mathrm{C}_{24}$ y el blindaje de $\mathrm{C}_{20}$ que de forma semejante pueden ser atribuidos respectivamente al efecto del átomo electronegativo en posición $\beta$ y a la interacción $\gamma$ gauche. Los corrimientos químicos de $\mathrm{C}_{20}, \mathrm{C}_{21} \mathrm{y}$ $\mathrm{C}_{25}$ permiten descartar, en concordancia con lo reportado ${ }^{17}$, la orientación axial de la función alcohólica.

La actividad estimuladora del crecimiento vegetal de los espirobrasinoesteroides (7) y (9) fue evaluada en diferentes ensayos biológicos y comparada con la del correspondiente espirobrasinoesteroide no funcionalizado en la posición $\mathrm{C}_{23}$ (4). Los resultados de los ensayos de inclinación de la lámina de 
arroz, el peso de los cotiledones del rábano y la longitud del hipocotilo del rábano realizados serán dados a conocer oportunamente. Se observó que los compuestos portadores de la funciones oxigenadas en la posición $\mathrm{C}_{23}$ muestran mayor actividad estimuladora del crecimiento vegetal que el compuesto no funcionalizado.

\section{PARTE EXPERIMENTAL}

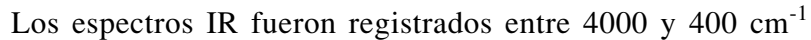
en un espectrómetro Phillips Analytical PU9800 FT-IR en pastillas de $\operatorname{KBr}\left(\right.$ Merck). Los espectros de $\mathrm{RMN}{ }^{1} \mathrm{H}$, y $\mathrm{RMN}{ }^{13} \mathrm{C}$ fueron registrados en un espectrómetro Bruker ACF-250, las mediciones se realizaron a temperatura ambiente en tubos de 5 $\mathrm{mm}$ de diámetro empleando como solvente $\mathrm{CDCl}_{3}$ (Merck Uvasol 99.8\%) y tetrametilsilano (TMS) como referencia interna. Las temperaturas de fusión fueron determinadas en capilares en un equipo Electrothermal 9100 y no están corregidas. Los productos fueron purificados haciendo uso de cromatografia de columna en gel de sílice 60 (Merck 70-230 mesh, ASTM). El curso de las reacciones fue controlado por cromatografia de capa delgada en cromatoplacas de gel de sílice (Merck) empleando como revelador una solución al $1 \%$ de vainillina en ácido perclórico al 50\%.

\section{(25R)-5 $\alpha$-Espirostan-2-eno (3)}

A $15 \mathrm{~g}$ (36 mmol) de tigogenina (2) disueltos en $100 \mathrm{~mL}$ de piridina seca se le añaden $10 \mathrm{~mL}$ de cloruro de mesilo. La solución se agita durante una hora y se vierte sobre agua/hielo/ $\mathrm{HCl}(500 \mathrm{~mL})$. El sólido se filtra y disuelto en $70 \mathrm{~mL}$ de $\mathrm{CHCl}_{3}$ se seca con $\mathrm{MgSO}_{4}$, se refluja media hora con carbón activado y el solvente se evapora a presión reducida. Al residuo disuelto en $200 \mathrm{~mL}$ de dimetilformamida seca, se le añaden $21 \mathrm{~g} \mathrm{de}$ $\mathrm{LiBr}$ y $17.5 \mathrm{~g}$ de $\mathrm{Li}_{2} \mathrm{CO}_{3}$ y se refluja en corriente de argón durante una hora. La mezcla se enfría y se vierte con agitación sobre solución acuosa de $\mathrm{HCl}$ al $10 \%$. El sólido se filtra, se lava con abundante agua, se seca en desecadora sobre pentóxido de fósforo y se purifica en columna cromatográfica utilizando como eluyente n-heptano/acetato de etilo 9/1. Rendimiento 10 g $(25 \mathrm{mmol}, 69 \%)$. Rf. 0.78 Sistema de solventes n-heptanol acetato de etilo (4/1). T. fusión 155-157. [lit $\left.{ }^{9} 155-156^{\circ} \mathrm{C}\right]$. IR $\left(v, \mathbf{c m}^{-1}\right): 3010\left(\mathrm{vCsp}_{2}-\mathrm{H}\right) ; 2954,2910,2890\left(\mathrm{vCsp}_{3}-\mathrm{H}\right) ; 1454$, $1375(\delta \mathrm{CH}) ; 1074,1052,1044(\mathrm{vC}-\mathrm{O}), 981,920,898,865$ (sist. espirocetálico). $\mathbf{R M N}{ }^{\mathbf{1}} \mathbf{H}(\delta, \mathbf{p p m}): 0.79$ (s, H-18); 0.77 (s, H-19); 0.96 (d, J=6.8 Hz, H-21); 0.77(d, J=6.8 Hz, H-27); 5.63 (m, H-2, H-3); 4.42 (m, H-16); 3.36/3.47 (m, H-26 a/e). $\mathbf{R M N}^{13} \mathbf{C}(\delta, \mathbf{p p m}): \mathrm{C}_{1} 39.8 ; \mathrm{C}_{2} 125.8 ; \mathrm{C}_{3} 125.8 ; \mathrm{C}_{4} 30.4 ; \mathrm{C}_{5}$ 41.4; $\mathrm{C}_{6} 28.7 ; \mathrm{C}_{7} 32.1 ; \mathrm{C}_{8} 34.8 ; \mathrm{C}_{9} 54.2 ; \mathrm{C}_{10} 35.8 ; \mathrm{C}_{11} 20.8$; $\mathrm{C}_{12} 40.2 ; \mathrm{C}_{13} 40.5 ; \mathrm{C}_{14} 56.4 ; \mathrm{C}_{15} 31.8 ; \mathrm{C}_{16} 80.9 ; \mathrm{C}_{17} 62.4 ; \mathrm{C}_{18}$ $16.4 ; \mathrm{C}_{19} 11.8 ; \mathrm{C}_{20} 41.7 ; \mathrm{C}_{21} 14.5 ; \mathrm{C}_{22} 109.2 ; \mathrm{C}_{23} 31.5 ; \mathrm{C}_{24}$ $28.9 ; \mathrm{C}_{25} 30.4 ; \mathrm{C}_{26} 66.8 ; \mathrm{C}_{27}$ 17.2.

\section{(25R)-5 $\alpha$-espirostan-2 $\alpha, 3 \alpha$-diol (3-epigitogenina) (4)}

A una disolución de $3 \mathrm{~g}(7.5 \mathrm{mmol})$ de la olefina (3) en tetrahidrofurano (THF) $(50 \mathrm{~mL})$, se le añaden $3 \mathrm{~g}$ de óxido de $\mathrm{N}$-metilmorfolina y $6 \mathrm{~mL}$ de una solución de $\mathrm{OsO}_{4}$ de concentración $12,5 \mathrm{mg} / \mathrm{mL}$ en terbutanol y se agita en atmósfera de argón durante 24 horas. Transcurrido este tiempo se añaden $6 \mathrm{~g}$ de $\mathrm{Na}_{2} \mathrm{SO}_{3}$ disueltos en $15 \mathrm{~mL}$ de agua y se agita durante dos horas, el THF se evapora y el residuo se extrae con acetato de etilo $(4 \times 50 \mathrm{~mL})$. La fase orgánica se lava con solución saturada de $\mathrm{NaCl}$, se seca con $\mathrm{MgSO}_{4}$ y el solvente se evapora a presión reducida. El sólido se recristaliza en acetona/n-heptano. Rendimiento $2.6 \mathrm{~g}$ (6.0 mmol, $80 \%)$; Rf. 0.39 Sistema de solventes $n$-heptano/acetato de etilo (4/1); T. fusión $264.7-266^{\circ} \mathrm{C}$; $\left[\right.$ lit $\left.^{9}{ }^{9} 263-264^{\circ} \mathrm{C}\right] . \mathbf{I R}\left(\mathrm{v}, \mathbf{~ c m}^{-1}\right): 3432(\mathrm{vOH}) ; 2952,2925\left(\mathrm{vCsp}_{3^{-}}\right.$ $\mathrm{H}) ; 1450,1376(\delta \mathrm{CH}) ; 1076,1052(\mathrm{vC}-\mathrm{O}), 981,920,898,865$ (sist. espirocetálico); $\mathbf{R M N}{ }^{\mathbf{1}} \mathbf{H}(\delta, \mathbf{p p m})$ : (ax./ec.) 1.22/1.68 (H1); 3.70 (dt J=4.0/11.8 Hz, H-2 ax.); 3.91 (m, H-3 ec.); 1.52 (H4 ec.); 1.48 (H-5 ax.); 1.30 (H-6 ec.); 0.92/1.70 (H-7); 1.50 (H8 ax.); 0.84 (H-9 ax.); 1.32/1.52 (H-11); 1.14/1.70 (H-12); 1.08 (H-14 ax.); 1.24/1.97 (H-15); 4.39 (m, H-16ax.); 1.76 (H-17 ax.); 0.76 (s, H-18); 0.81 (s, H-19); 1.88 (m, H-20); 0.96 (d, J=6.8 $\mathrm{Hz}, \mathrm{H}-21) ; 1.60$ (H-23 еc.); 1.45/1.62 (H-24); 1.63 (H-25 ax.); 3.36 (t, J=10.9 Hz H-26 ax.); 3.47 (dd, J=2.9/10.5 Hz, H-26 ec.); 0.79 (d, J=7.8 Hz, H-27); $\mathbf{R M N}{ }^{13} \mathbf{C}(\delta, \mathbf{p p m}): \mathrm{C}_{1} 40.4 ; \mathrm{C}_{2}$ $68.8 ; \mathrm{C}_{3} 69.0 ; \mathrm{C}_{4} 34.2 ; \mathrm{C}_{5} 38.5 ; \mathrm{C}_{6} 27.5 ; \mathrm{C}_{7} 31.9 ; \mathrm{C}_{8} 34.3 ; \mathrm{C}_{9}$ $54.1 ; \mathrm{C}_{10} 36.8 ; \mathrm{C}_{11} 20.6 ; \mathrm{C}_{12} 39.8 ; \mathrm{C}_{13} 40.4 ; \mathrm{C}_{14} 56.1 ; \mathrm{C}_{15} 31.5$; $\mathrm{C}_{16} 80.8 ; \mathrm{C}_{17} 62.0 ; \mathrm{C}_{18} 16.3 ; \mathrm{C}_{19} 12.3 ; \mathrm{C}_{20} 41.5 ; \mathrm{C}_{21} 14.3 ; \mathrm{C}_{22}$ 109.2; $\mathrm{C}_{23}$ 31.2; $\mathrm{C}_{24}$ 28.6; $\mathrm{C}_{25}$ 30.1; $\mathrm{C}_{26}$ 66.7; $\mathrm{C}_{27}$ 17.0.

\section{Diacetato de (25R)-5 $\alpha$-espirostan-2 $\alpha, 3 \alpha-\operatorname{diol}(5)$}

A $2 \mathrm{~g}(4.6 \mathrm{mmol})$ del diol (4) en piridina seca $(20 \mathrm{~mL})$ se le añaden $4 \mathrm{~mL}$ de anhidrido acético, la mezcla se agita por 24 horas, se vierte sobre mezcla agua/hielo/ $\mathrm{HCl}$, el sólido se filtra, se lava con agua y se recristaliza de acetona/n-heptano. Rendimiento $2.3 \mathrm{~g}$ (4.5 mmol, 98\%); Rf. 0.43 Sistema de solventes n-heptanolacetato de etilo (4/1); T. fusión $238-240.6^{\circ} \mathrm{C}$; IR $\left(v, \mathbf{c m}^{-1}\right): 2952,2945,2927,\left(v^{-C s p_{3}-H}\right) ; 1751,1741(v C=O)$; $1457,1367,(\delta \mathrm{CH}) ; 1255,1240$ (vC-O éster); 1057, $1038(\mathrm{v}$ C-O); 981, 920, 899 (sist. espirocetálico).

\section{(25R)-2 $\alpha, 3 \alpha$-Diacetoxi-5 $\alpha$-espirostan-23-ona (6)}

A $1 \mathrm{~g}(1.9 \mathrm{mmol})$ del diacetato $(5)$ disuelto en $20 \mathrm{~mL}$ de HOAc glacial se le añaden agitando $1.5 \mathrm{~mL}$ de trifluoruro de boro eterato $\left(\mathrm{BF}_{3} \cdot \mathrm{Et}_{2} \mathrm{O}\right)$ y $1.5 \mathrm{~g}$ de $\mathrm{NaNO}_{2}$ anhidro durante dos horas. Seguidamente se añaden $0.5 \mathrm{~mL}$ de $\mathrm{BF}_{3}$. $\mathrm{Et}_{2} \mathrm{O}$ adicionales y se agita durante 15 minutos. La mezcla se vierte sobre 100 $\mathrm{mL}$ de agua/hielo, se filtra, se disuelve en la mínima cantidad de benceno, se seca con $\mathrm{MgSO}_{4}$, se vierte en una columna con $60 \mathrm{~g}$ de alúmina de actividad Brockman III y se eluye con nheptano/acetato de etilo 3/2. El solvente se evapora al vácio y el producto se recristaliza de acetona/n-heptano. Rendimiento $0.7 \mathrm{~g}$, (1.32 mmol, 69\%); Rf. 0.35 Sistema de solventes $n$ heptano/acetato de etilo (4/1); T. fusión $235.1-236.7^{\circ} \mathrm{C}$; IR $\left(\mathrm{v}, \mathbf{c m}^{-1}\right): 2983,2964,2929,\left(\mathrm{vCsp}_{3}-\mathrm{H}\right) ; 1739(\mathrm{vC}=\mathrm{O}) ; 1456$, $1365(\delta \mathrm{CH}) ; 1261,1252$ (vC-O éster);1043, 1029 (vC-O); 964, 915 (sist. espirocetálico); $\mathbf{R M N}{ }^{1} \mathbf{H}(\delta, \mathbf{p p m}): 0.77$ (s, H-18); 0.89 (s, H-19); 0.93 (d, J=5.8 Hz, H-21); 0.90 (d, J=7.4 Hz, H27); 4.95 (ddd $\mathrm{J}=3.1 / 4.5 / 12.5 \mathrm{~Hz}, \mathrm{H}-2) ; 5.26$ (m, H-3); 4.60 (m, H-16 ); 2.89 (q, J=6.8 Hz, H-21); 2.42 (m, H-24); 2.29 (m, H-25); 3.78 (t, J=11.1 Hz, H-26 ax.); 3.58 (dd, H-26 ec.); 2.086 (s, $\mathrm{CH}_{3}$ acetato); $\mathbf{R M N}{ }^{13} \mathbf{C}(\delta, \mathbf{p p m}): \mathrm{C}_{1} 38.0 ; \mathrm{C}_{2} 70.0 ; \mathrm{C}_{3}$ 69.2; $\mathrm{C}_{4} 32.3 ; \mathrm{C}_{5} 39.3 ; \mathrm{C}_{6} 27.2 ; \mathrm{C}_{7} 31.8 ; \mathrm{C}_{8} 34.3 ; \mathrm{C}_{9} 54.1 ; \mathrm{C}_{10}$ $37.0 ; \mathrm{C}_{11} 20.7 ; \mathrm{C}_{12} 39.6 ; \mathrm{C}_{13} 41.0 ; \mathrm{C}_{14} 56.2 ; \mathrm{C}_{15}$ 31.6; $\mathrm{C}_{16}$ 83.2; $\mathrm{C}_{17}$ 61.7; $\mathrm{C}_{18} 16.2 ; \mathrm{C}_{19} 12.5 ; \mathrm{C}_{20} 34.7 ; \mathrm{C}_{21} 14.3 ; \mathrm{C}_{22} 109.8 ; \mathrm{C}_{23}$ 201.7; $\mathrm{C}_{24} 45.2 ; \mathrm{C}_{25}$ 35.8; $\mathrm{C}_{26} 65.6 ; \mathrm{C}_{27}$ 17.0; $\left(\mathrm{CH}_{3}\right.$ acetato $)$ 21.2, 21.0; $(\mathrm{C}=\mathrm{O}$ acetato $) 170.3$.

\section{(25R)-2 $\alpha, 3 \alpha$-dihidroxi-5 $\alpha$-espirostan-23-ona (7)}

A una solución de $200 \mathrm{mg}(0.377 \mathrm{mmol})$ de la cetona (6) y $0.3 \mathrm{~g}$ de $\mathrm{KOH}$ en metanol $(20 \mathrm{~mL})$ se refluja durante 30 minutos; la mezcla de reacción se deja enfriar y se concentra al vacio hasta la mitad del volumen inicial. Posteriormente se le añaden $60 \mathrm{~mL}$ de acetato de etilo y se lava con soluciones de $\mathrm{NaCl}$ saturada, $\mathrm{HCl} 5 \%$ y agua; el solvente se seca y se evapora al vacío. El producto se recristaliza de acetona/n-heptano. Rendimiento $156 \mathrm{mg}$ (0.349 mmol, 93\%); Rf. 0.32 Sistema de solventes n-heptano/acetato de etilo (1/1); T. fusión $249.6-251^{\circ} \mathrm{C}$; IR (v, $\left.\mathbf{c m}^{-1}\right): 3411,3384(\mathrm{vOH}) ; 2958,2927,2850\left(\mathrm{vCsp}_{3}-\mathrm{H}\right) ; 1734$ $(v \mathrm{C}=\mathrm{O}) ; 1456,1384(\delta \mathrm{CH}) ; 1049,1030(v \mathrm{C}-\mathrm{O}) ; 964$ (sist. espirocetálico); $\mathbf{R M N}{ }^{1} \mathbf{H}:(\delta, \mathbf{p p m}): 0.81$ (s, H-18) 0.76 (s, H-19); 0.93 (d, J=7.1 Hz, H-21); 0.93 (d, J=6.4 Hz, H-27); 
3.75 (m, H-2); 3.95 (m, H-3); 4.60 (m, H-16); 2.88 (q, H-20); 2.43 (dd, H-24); 3.78 (t, J=11.2 Hz, H-26 ax.); 3.58 (dd, H-26 ec.); $\mathbf{R M N}{ }^{13} \mathbf{C}(\delta, \mathbf{p p m}): \mathrm{C}_{1} 40.0 ; \mathrm{C}_{2} 69.0 ; \mathrm{C}_{3} 69 ; \mathrm{C}_{4} 34.2 ; \mathrm{C}_{5}$ $38.1 ; \mathrm{C}_{6} 27.5 ; \mathrm{C}_{7} 32.0 ; \mathrm{C}_{8} 34.4 ; \mathrm{C}_{9} 54.2 ; \mathrm{C}_{10} 37.0 ; \mathrm{C}_{11} 20.7 ; \mathrm{C}_{12}$ 39.7; $\mathrm{C}_{13} 41.0 ; \mathrm{C}_{14}$ 56.3; $\mathrm{C}_{15}$ 31.7; $\mathrm{C}_{16} 83.3 ; \mathrm{C}_{17}$ 61.7; $\mathrm{C}_{18}$ 16.2; $\mathrm{C}_{19} 12.5 ; \mathrm{C}_{20} 34.8 ; \mathrm{C}_{21} 14.4 ; \mathrm{C}_{22} 109.8 ; \mathrm{C}_{23} 202.0 ; \mathrm{C}_{24} 45.3$; $\mathrm{C}_{25} 35.8 ; \mathrm{C}_{26} 65.6 ; \mathrm{C}_{27}$ 17.1.

\section{(23S, 25R)-2 $\alpha, 3 \alpha$-diacetoxi-5 $\alpha$-espirostan-23-ol (8)}

A $350 \mathrm{mg}(0.660 \mathrm{mmol})$ de la cetona (6) en metanol (30 $\mathrm{mL})$ se le añaden agitando y manteniendo la temperatura bajo $10^{\circ} \mathrm{C}, 90 \mathrm{mg}(2.36 \mathrm{mmol})$ de $\mathrm{NaBH}_{4}$ en etanol $(5 \mathrm{~mL})$. La mezcla se agita 30 minutos, se vierte sobre solución saturada de $\mathrm{NaCl}$ y se extrae con acetato de etilo, se lava con agua, se seca con $\mathrm{MgSO}_{4}$ y el solvente se evapora al vacío. El producto se recristaliza de acetona/n-heptano. Rendimiento $300 \mathrm{mg}$ (0.563 mmol, $85 \%)$; Rf. 0.19 Sistema de solventes $n$-heptanol acetato de etilo (4/1); T. fusión $205.5-207.8^{\circ} \mathrm{C} ; \mathbf{I R}\left(\mathrm{v}, \mathbf{c m}^{-1}\right)$ : $3350(\mathrm{vOH}) ; 2953,2931\left(\mathrm{vCsp}_{3}-\mathrm{H}\right) ; 1747(\mathrm{vC}=\mathrm{O}) ; 1457,1371$ $(\delta \mathrm{CH}) ; 1251,1232(\mathrm{vC}-\mathrm{O}$ éster); 1054, 1052 (vC-O); 962 (sist. espirocetálico); $\mathbf{R M N}{ }^{\mathbf{1}} \mathbf{H}$ : $(\delta, \mathbf{p p m}): 0.81(\mathrm{~s}, \mathrm{H}-18) ; 0.89(\mathrm{~s}, \mathrm{H}-$ 19); 0.95 (d, J=6.5 Hz, H-21); 0.82 (d, J=9.0 Hz, H-27); 4.96 (m, H-2); 5.27 (m, H-3); 4.46 (m, H-16); 2.54 (q, J=6.8 Hz, H20); 3.25 (t, J=11.0 Hz, H-26 ax.) 3.46-3.38 (m, H-26 ec., H23 ax.); 2.09 (s, $\mathrm{CH}_{3}$ acetato); $\mathbf{R M N}{ }^{13} \mathbf{C}(\delta, \mathbf{p p m}): \mathrm{C}_{1} 38.0 ; \mathrm{C}_{2}$ 69.9; $\mathrm{C}_{3}$ 69.2; $\mathrm{C}_{4} 32.2 ; \mathrm{C}_{5} 39.6 ; \mathrm{C}_{6} 27.2 ; \mathrm{C}_{7} 31.8 ; \mathrm{C}_{8} 34.2 ; \mathrm{C}_{9}$ 54.1; $\mathrm{C}_{10} 37.0 ; \mathrm{C}_{11} 20.7 ; \mathrm{C}_{12} 39.9 ; \mathrm{C}_{13} 40.9 ; \mathrm{C}_{14}$ 56.0; $\mathrm{C}_{15} 31.6$; $\mathrm{C}_{16}$ 81.4; $\mathrm{C}_{17}$ 61.6; $\mathrm{C}_{18} 16.5 ; \mathrm{C}_{19} 12.4 ; \mathrm{C}_{20} 35.4 ; \mathrm{C}_{21} 14.0 ; \mathrm{C}_{22}$ $110.5 ; \mathrm{C}_{23}$ 66.9; $\mathrm{C}_{24} 38.4 ; \mathrm{C}_{25} 30.7 ; \mathrm{C}_{26} 65.8 ; \mathrm{C}_{27}$ 16.5; $\left(\mathrm{CH}_{3}\right.$ acetato) 21.1, 21.0; ( $\mathrm{C}=\mathrm{O}$ acetato $)$ 170.3.

\section{(23S, 25R)-5 $\alpha$-espirostan-2 $\alpha, 3 \alpha, 23$-triol (9)}

A $300 \mathrm{mg}(0.563 \mathrm{mmol})$ del diacetato $(\mathbf{8})$ disueltos en metanol $(20 \mathrm{~mL})$ se le añaden $0.3 \mathrm{~g}$ de $\mathrm{KOH}$. La mezcla se refluja durante 15 minutos, se añade $1 \mathrm{~mL}$ de ácido acético glacial y el solvente se elimina a presión reducida. El residuo se disuelve en acetato de etilo $(30 \mathrm{~mL})$ y la solución se lava con solución saturada de $\mathrm{Na}_{2} \mathrm{CO}_{3}(2 \times 10 \mathrm{~mL})$ y con agua $(5 \times 10 \mathrm{~mL})$ se seca con $\mathrm{MgSO}_{4}$ y el solvente se elimina a presión reducida. El producto se recristaliza de acetona. Rendimiento $224 \mathrm{mg}(0.500 \mathrm{mmol}$, $89 \%)$; Rf. 0.29 Sistema de solventes n-heptanolacetato de etilo (1/1); T. fusión $273.5-275^{\circ} \mathrm{C}$; IR ( $\left.\mathrm{v} \mathrm{cm}^{-1}\right)$ : $3378(\mathrm{vOH})$; 2954, 2927, $2873\left(\mathrm{vCsp}_{3}-\mathrm{H}\right)$; 1456, $1379(\delta \mathrm{CH}) ; 1095,1076,1054$ (vC-O); 993, 964, 939 (sist. espirocetálico).

\section{CONCLUSIONES}

- Fueron sintetizados dos nuevos análogos espirostánicos de brasinoesteroides portadores de funciones oxigenadas en la posición $\mathrm{C}_{23}$. Utilizando la tigogenina como material de partida es posible reproducir fácilmente las funciones características del anillo A de la 6-desoxocastasterona y posteriormente realizar la funcionalización de la posición $\mathrm{C}_{23}$ con rendimientos moderados.

- La funcionalización de la posición $\mathrm{C}_{23}$ produce cambios substanciales en las características espectroscópicas derivadas de la cadena lateral espirocetálica y no influye en las derivadas del núcleo esteroidal.

- Los resultados de la evaluación de la actividad biológica de los análogos sintetizados sugieren que la introducción de funciones oxigenadas en $\mathrm{C}_{23}$ produce un aumento de la actividad estimuladora del crecimiento vegetal.

\section{REFERENCIAS}

1. Michitada, K.; Mori, K.; Agric. Biol. Chem. 1983, 47, 97.

2. Sasse, J. M.; Physiol. Plantarum. 1990, 80, 401.

3. Okada, K.; Mori, K.; Agric. Biol. Chem. 1983, 47, 89.

4. Wei-Shan, Z.; Li-Zhoug, J.; Wei-Sheng, T.; A. Chim. Sin. 1988, 4, 332.

5. Kohout, L.; Strnad, M.; Collect. Czch. Chem. Comm. 1989, 54, 1019.

6. Kohout, L.; Velgova, H.; Strnad, M.; Collect. Czch. Chem. Comm. 1987, 52, 476.

7. Cerny, V.; Strnad, M.; Kaminek, M.; Collect. Czch. Chem. Comm. 1986, 51, 687.

8. Kohout, L.; Strnad, M.; Collect. Czch. Chem. Comm. 1986, 51, 447.

9. Coll, F.; Alonso, E.; Iglesias, M.; Marquardt, V.; Adam, G.; Rev. Cub. Quím. 1992, 6, 7.

10. Coll, F.; Alonso, E.; Adam, G.; Marquardt, V.; Patente Alemana 1989, C07J 63/00, 273638A.

11. Iglesias Artega, M. A.; Análogos Espirostánicos y Furostanicos de Brasinoesteroides. Tesis de Doctorado. Universidad de la Habana Cuba 1996.

12. Barton, D. H. R.; Sammes, P. G.; Taylor, M. V.; Westiuk, E.; J. Chem. Soc.(C) 1971, 1977.

13. Gonzalez, A. G.; Freire, R.; Garcia-Estrada, M. G.; Salazar, J. A.; Suarez, E.; Tetrahedron 1972, 28, 1289.

14. Fried, J.; Edwards, J. A.; Organic Reactions in Steroids Chemistry I. Van Nostrand Reinhold Co. N. Y. 1972, Capitulo. 2, p61.

15. Beckett, A. H.; Harper, N. J.; Balon, A. D. J.; Tetrahedron, 1959, 6, 319.

16. Mickova, R.; Collect. Czch. Chem. Comm. 1985, 50, 1239.

17. Agrawal, P. K.; Jain, D. C.; Gupta, R. K.; Thakur, R. S.; Phytochemistry 1985, 24, 2479. 